Estudios de

lingüística inglesa aplicada

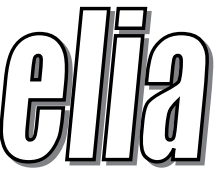

\title{
IDENTIFICACIÓN DE ERRORES PRAGMÁTICOS EN UN TEST ADAPTATIVO DE INGLÉS COMO LENGUA EXTRANJERA
}

\section{PRAGMATIC ERROR IDENTIFICATION IN A COMPUTER- ADAPTIVE TEST OF ENGLISH AS A FOREIGN LANGUAGE}

\author{
María Luisa Carrió Pastor \\ Universitat Politècnica de València, Spain \\ lcarrio@upv.es

\section{Beatriz Martín Marchante} \\ Universitat Politècnica de València, Spain \\ beamarm2@upvnet.upv.es
}

DOI: http://dx.doi.org/10.12795/elia.2016.i16.04

Este trabajo analiza las razones por las cuales un grupo de estudiantes falla las preguntas especificas que evalúan su competencia pragmalingüística en la prueba de dominio Oxford Online Placement Test (OOPT). El objetivo de este estudio es descubrir qué tipo de deficiencias en la competencia lingüística de estos estudiantes pueden estar implicadas en la producción de errores en estas preguntas, según su propia percepción y desde una perspectiva cognitiva y

ELIA 16, 2016, pp. 79-102～DOI: http://dx.doi.org/10.12795/elia.2016.i16.04 
metalingüística. Para ello, se diseñó un cuestionario de valoración restrospectivo que fue realizado por cuarenta y cuatro estudiantes de primer curso en la Facultat de Magisteri, Universitat de València (España) que debían acreditar tener un nivel B1 de lengua inglesa al acabar el curso. Los resultados indicaron que el motivo de error más frecuente en la parte de comprensión pragmática del OOPT era el desconocimiento de parte del vocabulario contenido en los diálogos que conformaban las preguntas correspondientes a esta parte de la prueba.

Palabras clave: pragmática, pruebas de lengua, inglés como lengua extranjera.

This paper analyses the reasons why a group of students fails the specific items that assess the pragmalinguistic competence in English in the Oxford Online Placement Test (OOPT). The work at hand aims to find out what deficiencies in their linguistic competence are involved in their production of errors in these items. The research has been carried out according to the students own perception and from a cognitive and meta-linguistic perspective. For this purpose, a retrospective questionnaire was designed and administered to forty-four students, taking an English course at the Facultat de Magisteri, Universitat de València (Spain). These students should certify a B1 level of English when finishing the course. The results showed that the most frequent source of errors in the pragmatic comprehension part of the OOPT was unawareness of some vocabulary contained in the dialogues corresponding to this part of the test.

Key words: pragmatics, language testing, English as a foreign language.

\section{Introducción}

La evaluación de los conocimientos lingüísticos de los aprendices de una lengua extranjera se ha investigado profusamente debido a la importancia de determinar tanto los niveles de aprendizaje como las necesidades para poder comunicarse de forma correcta y efectiva. Desde los años sesenta (Carroll, 1961), una de las mayores preocupaciones en el campo de la investigación sobre la evaluación de las pruebas de lenguas extranjeras ha sido capturar o recrear la esencia del uso de la lengua, de manera que resulten auténticas e integradoras. Muchos de estos estudios se han

ELIA 16, 2016, pp. 79-102～DOI: http://dx.doi.org/10.12795/elia.2016.i16.04 
centrado en aspectos puramente estructurales del lenguaje, como el uso del léxico o de la sintaxis, la morfología, la fonética, etc. (James, 1998; Carrió Pastor, 2005). Pero en las últimas décadas se ha prestado especial atención al efecto comunicativo del lenguaje y a los aspectos pragmáticos de la comunicación (Kasper, 2000; Rose y Kasper, 2001; Carrió-Pastor y Mestre, 2013ª ; Mestre y Carrió-Pastor, 2013a,b; y, Carrió-Pastor y Casas, 2015). El enfoque comunicativo sirvió de base para la investigación sobre la enseñanza de una lengua extranjera y dio lugar al surgimiento del Communicative Language Testing, lo cual implicaba la necesidad de evaluar la producción y la competencia lingüística. Gran parte del desarrollo de las pruebas de lengua se produjo como consecuencia directa de la perspectiva comunicativa en la enseñanza de lenguas presentada por investigadores tales como Savignon (1972), Candlin (1976), Widdowson (1978) y Johnson (1982), entre otros. Además, esta nueva concepción de la habilidad lingüística dio lugar a la creación de nuevos marcos de trabajo y modelos teóricos como el de Bachman (1990), Bachman y Palmer (1996) y Purpura (2004). En estos modelos, la habilidad lingüística abarca competencias múltiples, incluyendo en ella la competencia pragmática. La pragmática, según Leech (1974), estudia la relación entre las expresiones lingüísticas y sus usuarios, es decir, la relación entre el hablante y el contexto de la comunicación. Yule (1996: 3-4) la describe como:

La habilidad para tratar el significado comunicado por el hablante (o escritor) y el interpretado por el oyente o lector [...] e interpretar el significado intencional de la gente, sus (pre) supuestos, sus propósitos u objetivos, y el tipo de acción (por ejemplo peticiones) que el hablante lleva a cabo en la conversación.

Como explican Bachman y Palmer (1996: 90), la competencia pragmática "[...] incluye la competencia ilocutiva, o el conocimiento de las convenciones pragmáticas para realizar funciones del lenguaje aceptables y la competencia sociolingüística".

Bachman (1990) sostiene que los cuatro factores que pueden influir en los resultados de una prueba de lengua son: la habilidad comunicativa en una lengua; los aspectos del método; las características individuales, y 
las medidas de error aleatorias. Las características individuales incluirían:

1. Características personales (edad, sexo, nacionalidad, estatus de residente, lengua nativa, nivel y tipo de educación general, tipo y grado de preparación o experiencia anterior en una prueba dada).

2. Conocimiento del contexto.

3. Esquema afectivo.

4. Habilidad lingüística.

Según Bachman y Palmer (1996) la habilidad lingüística está constituida por dos componentes:

a. El conocimiento de la lengua que integra el conocimiento organizacional y el conocimiento pragmático que incluye el conocimiento funcional y sociolingüístico.

b. La competencia estratégica o estrategias metacognitivas.

Esta concepción de la habilidad lingüística pone de manifiesto la importancia que se ha concedido a la pragmática desde hace décadas, pero a pesar de los avances en éste ámbito, y sobre todo en el campo de la pragmalingüística, la evaluación de la competencia pragmática es un área compleja y difícil de acotar. Esto es debido a que algunas de sus unidades de estudio como las implicaturas, las fórmulas rutinarias o los actos de habla, al contrario que las de otras disciplinas lingüísticas como la sintaxis o la morfología, no están claramente definidas ni se pueden manipular analíticamente. Así pues, su sistematización continúa siendo muy complicada. Hemos de destacar que se han estudiado aspectos aislados de esta competencia dentro de la tradición de la teoría de los actos de habla (Austin, 1962; Searle, 1969) y de la cortesía (Brown y Levinson, 1987) a excepción de las pruebas de Walters (2007) y de Roever (2006, 2011: 469), como indica éste último investigador:

The main construct under investigation has been learners' ability to produce language that is appropriate and polite enough given the context (as defined by Brown \& Levinson, 1987), or their ability to judge whether exemplars of language are appropriate and polite in context. Productive studies have frequently employed DCTs, 
which elicit the target speech act with some external and internal modifications.

Por otra parte, hemos de destacar los estudios que se han realizado para la detección y clasificación de errores en la interlengua desde una perspectiva pragmática (Kasper, 2000; Rose y Kasper, 2001; BardoviHarlig, 2013), algunos de ellos se han hecho a través de un corpus de producción escrita (Carrió y Mestre, 2013b) o de exámenes online (Martín Marchante 2015).

Finalmente, incidimos en que el principal objetivo de este estudio a diferencia de otros anteriores, no es clasificar los errores pragmáticos, sino indagar sobre qué tipo de carencias, en su conocimiento de la lengua, da lugar a la producción de errores en la comprensión e interpretación las diferentes categorías pragmáticas incluidas en las preguntas del $O O P T$, según la percepción de los propios examinandos. Realizamos este planteamiento, ya que hemos observado que actualmente existe un vacío en cuanto a qué y cómo diagnosticar las deficiencias en la competencia pragmática de los aprendices de lengua extranjera. En este sentido, nuestra idea de partida es que se ha de incidir en los aspectos pragmáticos de la lengua desde el inicio de su proceso de adquisición, ya que son esenciales para poder dominarla y comprenderla. Entendemos que se podrían conocer las fortalezas y debilidades en la competencia pragmática partiendo de las percepciones de los propios estudiantes, a través de la autocorrección, de actividades de concienciación, preguntando en qué encuentran dificultad, o por qué piensan que tienen problemas en general o en particular.

\section{Método}

\subsection{Materiales}

Para llevar a cabo este estudio, se utilizaron los resultados obtenidos por cuarenta y cuatro estudiantes que realizaron el Oxford Online Placement Test $(O O P T)$. Esta es una prueba de dominio externa, que la Facultat de València facilita a los estudiantes para que puedan acreditar 
su nivel, facilitar la homologación y garantizar transparencia el proceso de clasificación de por tanto independiente del proceso de aprendizajeenseñanza. También se utilizó un cuestionario de valoración retrospectivo que se describe más adelante. Los participantes estudiaban el primer curso en el Grado de Magisterio en la Universitat de València y estaban matriculados en la asignatura obligatoria de seis créditos LLengua estrangera per a mestres: anglès. Esta asignatura tiene dos partes, una de carácter lingüístico y otra de carácter didáctico. La asignatura persigue dos objetivos, por una parte que los estudiantes consigan un nivel B1 del Marco Común Europeo de Referencia para las Lenguas (MCERL) y por otra, deben adquirir conocimientos que les capaciten para impartir clases con un enfoque metodológico de Aprendizaje Integrado de Contenidos y Lenguas Extranjeras (AICLE). Los estudiantes realizaron las pruebas de dominio externas (OOPT) en enero del 2014, coincidiendo con la finalización de la asignatura y el examen escrito de la misma.

El objetivo principal del OOPT es medir la competencia comunicativa de los examinandos y no ceñirse a evaluar únicamente los conocimientos gramaticales o léxicos. Purpura (2009) explica que esta prueba mide la habilidad para entender las formas gramaticales en distintos contextos y el significado de éstas, midiendo también en qué grado los aprendices son capaces de utilizar estos recursos lingüísticos en inglés adaptándose a cada situación, matizando que en el desarrollo de este test se tuvieron en cuenta los descriptores del Marco Común Europeo (2001) así como las tendencias actuales en investigación referentes a la naturaleza de la competencia comunicativa y el aprendizaje de lenguas extranjeras.

El OOPT está compuesto de dos partes, una que evalúa el uso del inglés y la otra que valora la comprensión oral. La primera contiene treinta preguntas (aunque puede variar) y evalúa el vocabulario, la gramática y la comprensión semántica en una conversación. Dentro de esta parte, la sección denominada Use of English incluye un sub-test de pragmática ya que, como afirma Purpura (2009), es importante que tengamos información sobre la habilidad gramatical y pragmática de los aprendices si queremos que mejoren en este aspecto. A continuación mostramos un ejemplo de este tipo de preguntas en la Figura 1: 


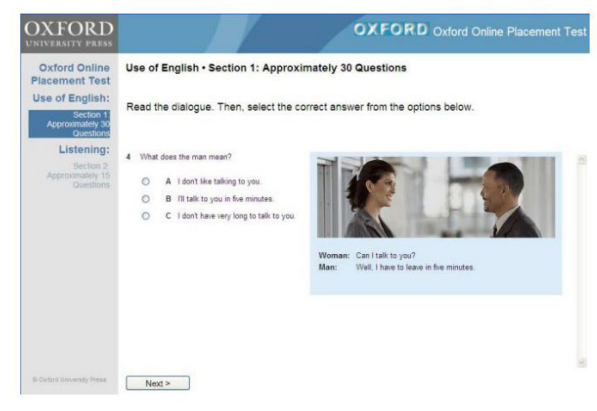

Figura 1. Muestra de significado implícito en el $O O P T$

En este ejemplo, en la fotografía una mujer pregunta si puede hablar con un hombre. Él contesta que solo tiene cinco minutos, indicándole así que la conversación se tiene que limitar a cinco minutos. El significado en este contexto es que sí puede hablar, pero este significado es implícito más que explícito. Diciendo que tiene que irse en cinco minutos, lo que desea comunicar es: "Sí, hablemos, pero solo puedo hablar cinco minutos".

La segunda parte, de comprensión oral, contiene quince preguntas y evalúa también la comprensión del significado en la conversación. El test se puntúa globalmente pero también desglosa la puntuación de la sección de uso del inglés y la de comprensión oral. Además, informa del tiempo dedicado a cada una de ellas. La puntuación se muestra al estudiante en una escala del 0 al 120, dando el mismo peso a las dos secciones. Junto con la puntuación aparecen los descriptores de conocimiento y uso de la lengua. Para este estudio se seleccionaron examinandos cuyo objetivo era poder superar el test para obtener un nivel B1, siguiendo el Marco Común Europeo (2001). Así, cuando la puntuación de un examinando se situaba dentro del rango 40-60, su nivel correspondiente era un B1 o preintermedio.

El cuestionario de valoración retrospectiva (véase Anejo 1) incluyó un espacio para el nombre y el grupo al que el encuestado pertenecía, así como instrucciones para su cumplimentación. Se plantearon dos tipos de preguntas: una pregunta cerrada y otra abierta. La pregunta cerrada era de 
tipo elección múltiple, con seis opciones. El diseño del cuestionario se basó en las anotaciones registradas en el diario de la profesora durante entrevistas individuales realizadas por motivo de las revisiones de los exámenes y antes de realizar una clasificación de las preguntas de pragmática, lo cual es el objetivo de otro estudio. En las entrevistas la profesora preguntó a cada uno de los alumnos si entendían la frase o texto contenidos en cada pregunta errada. Si el examinando contestaba afirmativamente se le pedía que tradujese dicho texto para comprobar que efectivamente había entendido la pregunta. En muchas ocasiones se comprobó que en realidad no era así. Si la respuesta era "no", entonces se le pedía que identificase la parte o partes de la pregunta que no entendía y que explicase cuál era el motivo por el que no entendía. Todas las observaciones se anotaron en un "diario de profesor" y sirvieron para definir las seis opciones que en el cuestionario de valoración aparecen adaptadas para facilitar a los alumnos su compresión, por ejemplo: "no entendí nada" en lugar de "falta de comprensión del texto en general". Se asignó una letra a cada categoría de respuesta, para identificar la causa de que no lo cumplimentaran correctamente, entre las que se incluyó: ansiedad, lapsus, falta de comprensión del texto, es decir, incomprensión general (o total) del diálogo, incomprensión de una o dos palabras, incomprensión de tres o más palabras, interpretación incorrecta aun conociendo el vocabulario del mismo, y finalmente, la opción "otros" para que los estudiantes pudieran explicar algunas otras razones que no apareciesen entre las seis opciones.

El cuestionario contenía una pregunta de respuesta abierta para permitir que los encuestados pudieran aclarar su respuesta y subsanar de esta manera cualquier fallo, tanto en el planteamiento como en la pregunta cerrada. Ambos tipos de preguntas proporcionaron información sobre la opinión o percepción de los informantes acerca de los motivos por los que erraron las preguntas relacionadas con aspectos pragmáticos y que se centraban en el rango de las preguntas 11 al 20 de la prueba OOPT.

\subsection{Proceso del estudio}

Una vez realizadas las pruebas de nivel por los alumnos, se recabó la información necesaria para determinar los motivos por los que los alumnos 
habían fallado determinadas preguntas de la prueba $O O P T$ a través de un "diario de profesor".

Los resultados de las pruebas del OOPT realizadas por los estudiantes se extrajeron a través de la sección Markbook de la página web del $O O P T$ de manera inmediata. Estos resultados se exportaron a una hoja de cálculo. Posteriormente, tras comunicar los resultados a todos los estudiantes, se convocó a un grupo seleccionado de 44 de ellos a una sesión de revisión de la prueba de nivel. La muestra fue homogénea en cuanto a edad, entre 18 y 25 años, y nivel de inglés ya que todos oscilaban entre A2 y B1, niveles adquiridos a través de formación académica reglada. Cuatro de los participantes eran hombres y 40 eran mujeres, por lo que podemos afirmar que en este estudio las variables antropométricas tienen poca variabilidad. Se explicó a los alumnos en qué consistía el cuestionario insistiendo en el hecho de que responder al cuestionario les ayudaría a reflexionar sobre su propio aprendizaje ya que en las respuestas que diesen hallarían los motivos de los fallos que habían cometido durante la prueba, así como las claves para mejorar.

Durante la sesión de revisión se entregó a cada alumno el cuestionario retrospectivo y se les recordó que solo se utilizaría con las preguntas de pragmática. Los alumnos consultaron en sus respectivos ordenadores los resultados mostrados a través de un resumen con la ayuda, en ocasiones, de las profesoras. Cuando el alumno revisaba la pregunta de pragmática incorrecta pasaba a responder el cuestionario, en el que marcaba con una "x" en la casilla correspondiente al motivo por el cual había errado la pregunta. Al finalizar las revisiones de las pruebas se recopilaron un total de 44 cuestionarios.

Para establecer las relaciones existentes entre las preguntas y los motivos por los que se erraron se realizó en primer lugar una tabla de contingencia con el número de individuos que contestaron incorrectamente cada cuestión según el motivo de error clasificado bajo la siguiente categorización en el cuestionario retrospectivo:

a) Ansiedad, lapsus

b) Incomprensión general del texto 

c) Incomprensión de 1 o 2 palabras del texto
d) Incomprensión de 3 o más palabras del texto
e) Interpretación incorrecta del texto aun conociendo el vocabulario del mismo
f) Otros

En segundo lugar se desarrolló un análisis factorial de correspondencias para describir las relaciones existentes entre las preguntas del bloque de pragmática y los motivos de error, así como entre las propias categorías de cada una de estas dos variables (preguntas entre sí y motivos entre sí). A partir de la tabla de contingencia original, se construyó otra que constituyó el input para el desarrollo del análisis, donde para cada pregunta y motivo se calculó el porcentaje de individuos que erraron por ese motivo sobre el total de individuos que fallaron.

\section{Resultados}

A continuación se presentan los resultados del análisis de las razones expuestas en el cuestionario realizado por los alumnos que asistieron las revisiones de exámenes tras finalizar la prueba $O O P T$. Para describir las relaciones existentes entre las preguntas que evalúan la competencia pragmática (de la número 11 a la 20) y los motivos por los que los examinandos las yerran se ha disgregado para cada pregunta el porcentaje de error manteniendo la base de 44 examinandos. Seguidamente, la Tabla 1 muestra la distribución porcentual de motivos de fallos para cada una de las preguntas pragmáticas (input del subsiguiente análisis de correspondencias):

$\begin{array}{llllllllll}\mathrm{P} 11 & \mathrm{P} 12 & \mathrm{P} 13 & \mathrm{P} 14 & \mathrm{P} 15 & \text { P16 } & \text { P17 } & \text { P18 } & \text { P19 } & \text { P20 }\end{array}$

$\begin{array}{lllllllllll}\mathrm{N}= & 21 & 25 & 22 & 26 & 26 & 25 & 27 & 20 & 17 & 21\end{array}$

ELIA 16, 2016, pp. 79-102～DOI: http://dx.doi.org/10.12795/elia.2016.i16.04 
Ansiedad, lapsus $\quad 4,8 \quad 8,0 \quad 13,6 \quad 15,4 \quad 3,8 \quad 24,0 \quad 18,5 \quad 15,0 \quad 11,8 \quad 0,0$

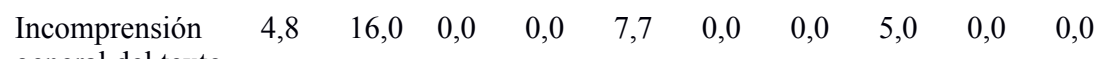
general del texto

Incomprensión $\quad 42,9 \quad 28,0 \quad 40,9 \quad 42,3 \quad 42,3 \quad 36,0 \quad 29,6 \quad 45,0 \quad 17,6 \quad 52,4$ 1-2 palabras

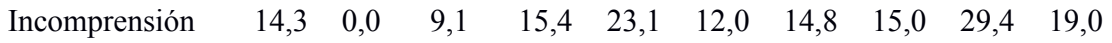

$3+$ palabras

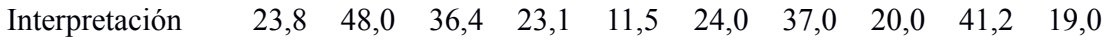
incorrecta aun conociendo vocabulario

Otros

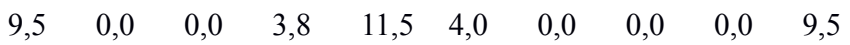

TOTAL $\begin{array}{llllllllll}100 & 100 & 100 & 100 & 100 & 100 & 100 & 100 & 100 & 100\end{array}$

Tabla 1. Porcentaje de motivos de error en las preguntas del bloque. Base: sujetos que han errado cada pregunta

La Figura 2 permite visualizar con mayor claridad la similitud entre preguntas en cuanto a la distribución de las razones de las preguntas contestadas incorrectamente:

ELIA 16, 2016, pp. 79-102ＤOI: http://dx.doi.org/10.12795/elia.2016.116.04 


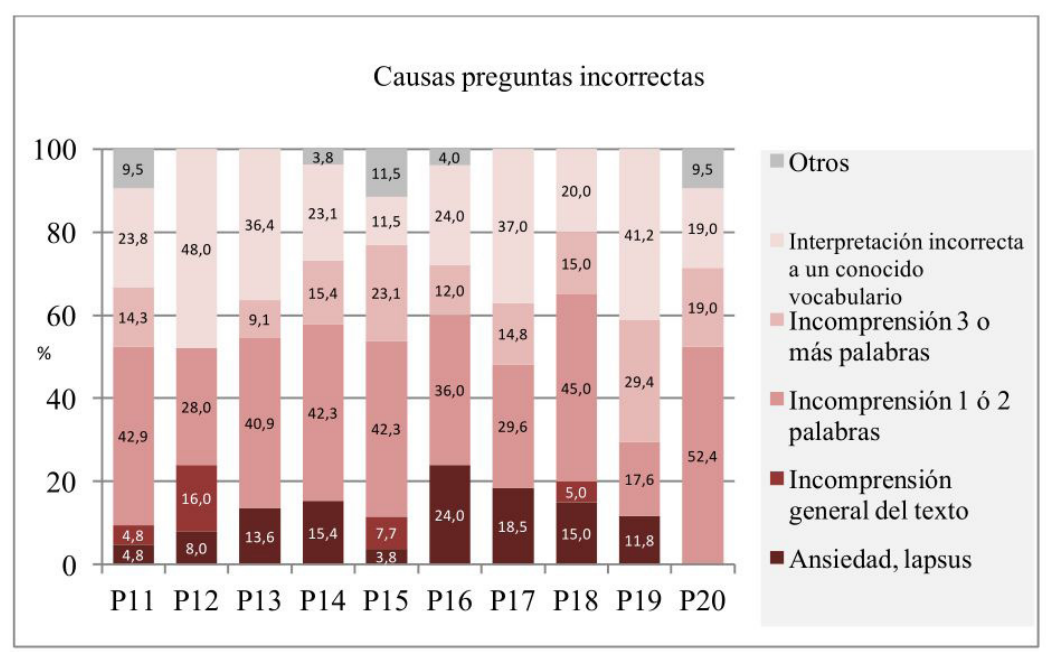

Figura 2. Causas de preguntas incorrectas

Se observa cómo la incomprensión de una o dos palabras es el motivo de fallo que con más frecuencia han alegado los examinandos, ya que se repite en siete de las diez preguntas. Las únicas preguntas en las que el motivo de fallo en pragmática es otro son las P12, P17 y P19. En estos tres casos, la causa que alegaron los examinandos fue la interpretación incorrecta del diálogo aun siendo conocedores del vocabulario. La respuesta 'Otros' se citó en algunas preguntas (P11, P15 y P20) con una mínima frecuencia. Se consideró que podía ser complicado explicar el significado de una dimensión dominada por esta categoría que agrupa motivos de diferente índole. Se propuso, por tanto, desarrollar un modelo que evitase el posible efecto negativo de esta categoría. El modelo que se expone en la Tabla 2 analiza la matriz de motivos de fallo, una vez excluido el apartado 'Otros':

$\begin{array}{lcccccccccc} & \mathrm{P} 11 & \mathrm{P} 12 & \mathrm{P} 13 & \mathrm{P} 14 & \mathrm{P} 15 & \mathrm{P} 16 & \mathrm{P} 17 & \mathrm{P} 18 & \mathrm{P} 19 & \mathrm{P} 20 \\ \mathrm{~N}= & 19 & 25 & 22 & 25 & 23 & 24 & 27 & 20 & 17 & 19 \\ \begin{array}{l}\text { Ansiedad, } \\ \text { lapsus }\end{array} & 5,3 & 8,0 & 13,6 & 16,0 & 4,3 & 25,0 & 18,5 & 15,0 & 11,8 & 0,0\end{array}$

ELIA 16, 2016, pp. 79-102～DOI: http://dx.doi.org/10.12795/elia.2016.i16.04 


\begin{tabular}{|c|c|c|c|c|c|c|c|c|c|c|}
\hline $\begin{array}{l}\text { Falta } \\
\text { comprensión } \\
\text { general }\end{array}$ & 5,3 & 16,0 & 0,0 & 0,0 & 8,7 & 0,0 & 0,0 & 5,0 & 0,0 & 0,0 \\
\hline $\begin{array}{l}\text { Incomprensión } \\
1-2 \text { palabras }\end{array}$ & 47,4 & 28,0 & 40,9 & 44,0 & 47,8 & 37,5 & 29,6 & 45,0 & 17,6 & \\
\hline $\begin{array}{l}\text { Incomprensión } \\
3+\text { palabras }\end{array}$ & 15,8 & 0,0 & 9,1 & 16,0 & 26,1 & 12,5 & 14,8 & 15,0 & 29,4 & \\
\hline $\begin{array}{l}\text { Interpretación } \\
\text { incorrecta aun } \\
\text { conociendo } \\
\text { vocabulario }\end{array}$ & 26,3 & 48,0 & 36,4 & 24,0 & 13,0 & 25,0 & 37,0 & 20,0 & 41,2 & \\
\hline TOTAL & 100 & 100 & 100 & 100 & 100 & 100 & 100 & 100 & 100 & 100 \\
\hline
\end{tabular}

Tabla 2. Porcentaje de motivos de fallos en las preguntas del bloque, excluyendo 'Otros'.

A continuación, se observa en la Tabla 3 que el primer resultado destacable es la confirmación de que ambas variables (preguntas y motivos de fallos) no son aspectos independientes $\left(\mathrm{Chi}^{2}=224,9 ; \mathrm{p}<0,001\right)$. Es decir, la distribución de motivos a lo largo de las distintas preguntas no puede aceptarse como similar, ya que los alumnos fallan unas preguntas por determinados motivos que son diferentes a otros. La variabilidad explicada por la primera dimensión es el $42,5 \%$ de la total y por la segunda el $33,9 \%$ de la total. Acumulan, pues, un 76,5\% del total. (Tabla 3):

\begin{tabular}{lcccccc} 
& Valor & & \multicolumn{2}{c}{ Chi- } & \multicolumn{2}{c}{ Proporción de inercia } \\
Dimensión & propio & Inercia & cuadrado & Sig & Explicada & Acumulada \\
\hline 1 &, 309 &, 096 & & &, 425 &, 425 \\
2 &, 276 &, 076 & & &, 339 &, 765 \\
3 &, 185 &, 034 & & &, 152 &, 917 \\
4 &, 137 &, 019 & & &, 083 & 1,000 \\
Total & &, 225 & 224,956 &, 000 & 1,000 & 1,000
\end{tabular}

Tabla 3. Dimensiones y variabilidad

Es decir, la Tabla 3 muestra una variabilidad (es decir, unas combinaciones de pregunta-error son más frecuentes que otras) y el modelo estadístico ha sido capaz de explicar parte de ella.

ELIA 16, 2016, pp. 79-102～DOI: http://dx.doi.org/10.12795/elia.2016.i16.04 
El modelo de análisis de correspondencias que se aprecia en la Figura 3 trata de visualizar gráficamente esas asociaciones entre los motivos de fallo pragmático y las preguntas. La idea es que aquellas preguntas en las que se tiende a fallar por un motivo concreto aparecen en la Figura 3 más próximas entre sí y más alejadas del resto. Es un mapa construido a partir de la cantidad de veces que se da un tipo de error en una pregunta. Para la representación, el modelo matemático utiliza dos ejes que básicamente son dos direcciones imaginarias que oponen preguntas asociadas a fallos de causas distintas. En principio, dos dimensiones son suficientes para describir y representar las asociaciones entre las categorías de las variables. Cuanta más independencia hay entre las preguntas (en el sentido de que cada una de ellas se falla exclusivamente por una causa), más dimensiones son necesarias. El mapa de posicionamiento resultante es el que sigue (Figura 3):

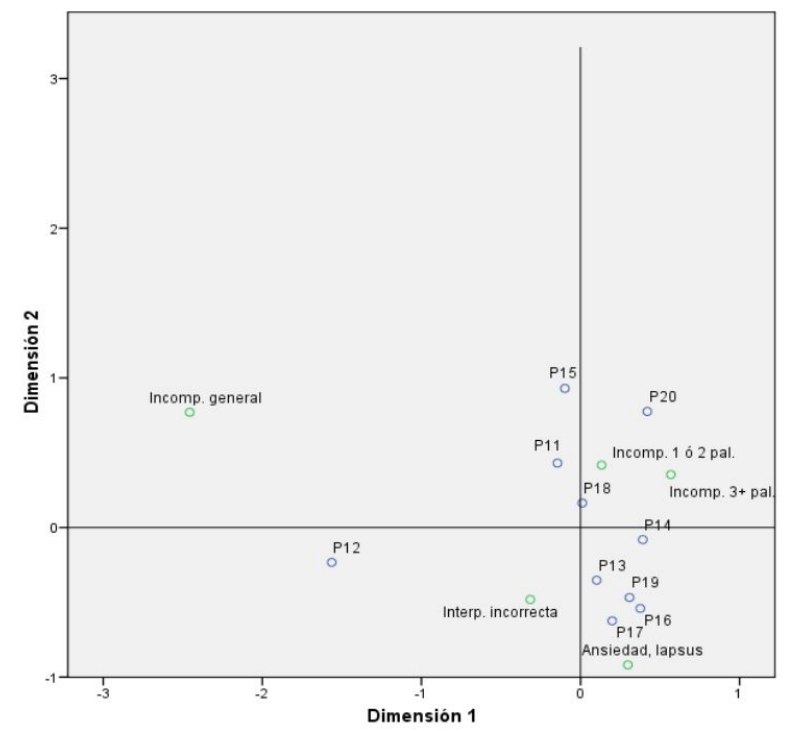

Figura 3. Mapa de asociaciones entre las categorías de las variables

La dimensión 1 separa fundamentalmente aquellas preguntas que se han fallado por incomprensión general del texto de aquellas que 
se han fallado debido a otros motivos. La figura presenta una estructura ciertamente asimétrica por la importancia discriminante de ese motivo. Si observamos la Tabla 2, en la P12, un 16,0\% de los alumnos que erraron por motivo definido, lo hicieron porque 'no entendieron nada'. En el resto de preguntas, el peso de este aspecto es muy inferior, de hecho es nulo en 6 de las preguntas. Básicamente, el análisis nos dice que la P12 es diferente a las demás; ya que se falla por una mayor incomprensión que el resto.

La dimensión 2, por otra parte, separa las preguntas que se fallaron por motivos relacionados con la incomprensión (parte superior) frente a las que se fallaron por ansiedad, lapsus o una interpretación incorrecta. Este tipo de mapas enfatizan las diferencias entre las categorías de una variable a través de la otra.

La P12 es la más asociada a errores por incomprensión general; aunque también aparece moderadamente asociada a errores relacionados a una interpretación incorrecta. Es decir, un 16\% de los errores cometidos en la P12 son por una falta general de comprensión de la pregunta y este porcentaje es más elevado que en cualquier otra pregunta. Esto no significa que la incomprensión general del texto sea el motivo de fallo más recurrente en la $\mathrm{P} 12$, pero sí que su peso es mayor que en el resto de preguntas. De hecho, en la P12 el motivo más habitual es la interpretación incorrecta $(48,0 \%)$. Como este porcentaje es más elevado que en el resto de las preguntas, el mapa tiende a aproximar estos dos elementos.

Las P13, P16, P17 y P19 son bastante similares entre sí, en cuanto a la distribución de los tipos de fallos cometidos. Los fallos en estas preguntas tienen que ver con el nerviosismo de los alumnos o también con no haber sabido interpretar bien lo que se preguntaba. Es decir, se sitúan en una zona común del mapa anterior, lo que significa que la asociación pregunta-fallo es frecuente en la muestra. En efecto, en la Tabla 2 observamos que un $13,6 \%$ de fallos en la P13 son por ansiedad, un $25,0 \%$ en la P16, un $18,5 \%$, en la P17 y un $11,8 \%$ en la P19. Obsérvese que son porcentajes, en general, más elevados que la media general.

La P14 está relativamente asociada a la incomprensión de tres o 
más palabras. Esta pregunta también tiene un porcentaje significativo de fallos $(16,0 \%)$ por ansiedad, pero como la incomprensión de 1-2 palabras $(44,0 \%)$ es más alta que en el resto, aparece desplazada en el mapa hacia la parte superior.

La P18 se ubica en una zona del mapa próxima al origen, lo que quiere decir que exhibe un perfil en la media general, y no se asocia especialmente a un motivo de fallo que la diferencie del resto. Las P11, P15 y P20 son preguntas que se sitúan en el área de las preguntas donde no se comprendieron unas cuantas palabras ( 1 o 2). En efecto, el porcentaje que supone este tipo de fallo es de $47,4 \%, 47,8 \%$ y $57,9 \%$ respectivamente, más elevado que en el resto de preguntas (Véase anejo 2).

En la Figura 4 se muestra un ejemplo de los resultados obtenidos en el ítem 15.

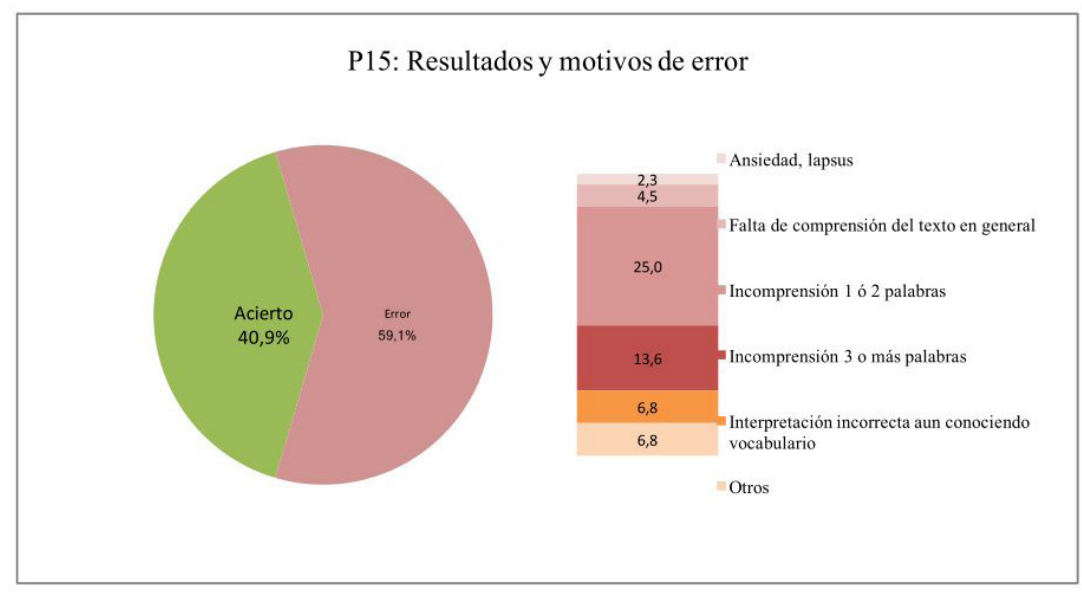

Figura 4. Porcentajes de examinandos y motivos de error en la pregunta 15

El $59,1 \%$ de los examinandos no contestaron correctamente la pregunta 15 siendo la incomprensión de una o dos palabras la razón más común. A continuación se expone un ejemplo de la pregunta 15 clasificada 
como inferencia trópica lexicalizada, siguiendo a Gallardo $(2006)^{1}$ que incluye elementos tales como dichos, refranes, fórmulas o rutinas, etc.

Ejemplo 1. ITL (Idiom, frase idiomática)

Man: I'm going to throw my hat in the ring for the Managing Director job.

What do you think?

Woman: Well, to be honest, I think it's a bit of a long shot.

What does the man mean?

A. I'm going to resign from the Managing Director job.

$B$. I'm going to apply for the Managing Director job.

C. I 'm going to accept the Managing Director job.

Obsérvese la frase idiomática: to throw my hat in the ring, es decir "lanzarse al ruedo". El 45,8\% de las muestras correspondientes al ítem 15 resultaron ser inferencias trópicas lexicalizadas; el 12,5\% fueron actos de habla; el $29,2 \%$, actos de habla indirectos y el $12,5 \%$ resultaron ser implicaturas.

\section{Conclusiones}

Los resultados obtenidos tras el análisis factorial de correspondencias indican que el motivo "incomprensión de una o dos palabras", es el que con más frecuencia han alegado los examinandos ya que se repite en siete de las diez preguntas que conforman el bloque de pragmática. Las preguntas se fallan más frecuentemente por la incomprensión de una o dos palabras, con un porcentaje que oscila entre el $28,0 \%$ y el $52,4 \%$.

1 Gallardo (1996) define las "implicaturas" (Grice, 1975) como un tipo de significado inferencial no convencional no basado en el uso de ciertas palabras, sino en la aplicación de ciertas normas comunicativas. Tienen carácter social y quedan fuera de las estructuras del lenguaje (son las inferencias). El significado que no se dice pero se infiere de un enunciado es una implicatura, mientras que las implicaciones, matiza la autora, suelen formar parte del significado léxico de las palabras y se sitúan en el terreno de la semántica.

ELIA 16, 2016, pp. 79-102

DOI: http://dx.doi.org/10.12795/elia.2016.i16.04 
En las P19, P12, P17 se han de destacar los fallos por la interpretación incorrecta del dialogo, aun cuando conocen el vocabulario y el rango de porcentaje se encuentra entre un $11,5 \%$ y un $48,0 \%$. Se ha detectado en la P12 que la incomprensión general del diálogo contenido en la pregunta es una causa con un peso relativamente elevado en comparación con el resto de preguntas. Por lo tanto, observamos que es difícil para este grupo de estudiantes comprender algunos de los diálogos y realizar inferencias debido a la falta de vocabulario.

Es evidente que la falta de vocabulario repercute en la comprensión lectora y por lo tanto en su capacidad de comunicación. Asimismo, el nivel de lectura se ha de tener en cuenta al evaluar la competencia pragmática, ya que como indica Coady (1979, citado en Clapham 1996), un nivel bajo de comprensión lectora impide el uso de habilidades como la inferencia y la predicción y produce incapacidad para decodificar el lenguaje. Contrariamente, a medida que los aprendices adquieren mayor conocimiento y uso, cada vez ponen menos énfasis en el proceso de decodificación y más en las estrategias cognitivas. También los fallos semánticos y de acceso léxico se deben, según Gallardo (2005), al déficit en la construcción de los actos de habla proposicionales o de estructuras sintácticas cohesionadas, como demuestran estudios llevados a cabo con hablantes con anomía o pobreza léxica. La investigación en el área de la psicología cognitiva ha puesto mucho énfasis en las habilidades de reconocimiento de palabras, conocimiento del vocabulario o procesamiento estratégico y este hecho ha influido en la forma en cómo la pragmática se describe teóricamente.

A raíz de los resultados extraídos, creemos que se debería de profundizar desde los primeros estadios de aprendizaje de una lengua extranjera en la enseñanza del léxico y su significado. Se trataría de aspirar a la enseñanza del vocabulario no tanto como conjunto de palabras, sino desde la perspectiva de la pragmática léxica. Para ello se pueden diseñar materiales e integrar programas que ayuden a mejorar las habilidades pragmáticas de los alumnos. Sería conveniente, por tanto, que el constructo se definiera dentro de un contexto verdaderamente léxico e integrando el significado pragmático. 
Sintetizando, sugerimos que si uno de los objetivos de las pruebas estandarizadas e informatizadas (adaptativas o no) es evaluar la pragmática enunciativa, la selección y diseño de las preguntas relacionadas con el significado no convencional (implicaturas conversacionales generalizadas y anómalas) se debería complementar con pruebas orales que permitan la interacción de los examinandos. De esta manera, la prueba no se limita a la evaluación de la comprensión de actos de habla y rutinas, sino también a la evaluación de la capacidad del aprendiz para producir estas unidades pragmáticas en contextos específicos. Asimismo, creemos que la inclusión de preguntas que contengan una interpretación pragmática conviene realizarse en formato video para que pueda producirse una interacción completa entre emisor y receptor, integrándose un aspecto tan importante como es la expresión corporal, ya que como afirma Abercrombie (1968: 55) "We speak with our vocal organs, but we converse with our whole body". Por último entendemos que estas soluciones pueden resultar de difícil aplicación en algunos sistemas de enseñanza y contextos académicos. Sin embargo, sí vemos factible y necesaria una cuidada selección de las preguntas diseñadas para evaluar la competencia pragmática que se incluyen en las pruebas estandarizadas, sean adaptativas o no. Independientemente de la necesidad indudable de que nuestros estudiantes reciban instrucción en aspectos pragmalingüísticos y léxico-semánticos de la lengua extranjera, es imprescindible que las preguntas de pragmática del OOPT analizadas en este trabajo deben mejorar su calidad disminuyendo en algunos casos, su índice de dificultad y optimizando su índice de discriminación.

\section{Referencias bibliográficas}

Abercrombie, D. (1968). Paralanguage. International Journal of Language \& Communication Disorders, 3(1), 55-59. https://doi. org/10.3109/13682826809011441

Alderson, J. C. (2000). Technology in testing: The present and the future. System, 28(4), 593-603. https://doi.org/10.1016/S0346-251X(00)00040-3

Austin, J. L. (1962). How to Do Things with Words. Oxford: England Calderon Press.

ELIA 16, 2016, pp. 79-102～DOI: http://dx.doi.org/10.12795/elia.2016.i16.04 
Bachman, L. F y Palmer, A. S. (1996). Language Testing in Practice. Oxford: University Press Oxford.

Bachman, L. F. (1990). Fundamental considerations in language testing. Oxford: Oxford University Press.

Bardovi-Harlig, K. (2013). Developing L2 pragmatics. Language Learning, 63(1), 68-86. https://doi.org/10.1111/j.1467-9922.2012.00738.x

Brown, P. y Levinson, S. D. (1987). Politeness: some universals in language usage. Cambridge: Cambridge University Press.

Candlin, C. N. (1976). Communicative language teaching and the depth to pragmatics, en C. Rameh (ed.), Georgetown University Roundtable Washington: Georgetown University Press.

Carrió Pastor, M. L. (2005). Contrastive analysis of scientific-technical discourse: common writing errors and variations in the use of English as a nonnative language. Ann Arbor: University of Michigan Press.

Carrió-Pastor, M. L. y Casas, M. (2015). The Use of Corpora to Identify the Pragmatic Knowledge Associated with Different Levels of Language Proficiency. En S. Gesuato, Bianchi, F. y W. Cheng (Eds.) Teaching, Learning and Investigating Pragmatics (pp. 207-230). Cambridge: Cambridge Scholars.

Carrió-Pastor, M. L. y Mestre Mestre, E. M. (2013a). Increasing pragmatic awareness in the L2 classroom. En J. W. Schwieter (Ed.) Studies and Global Perspectives of Second Language Teaching and Learning (pp. 241 - 264). New York: Information Age Publishing.

Carrió-Pastor, M. L. y Mestre, E. (2013b). A proposal for the tagging of grammatical and pragmatic errors. Research in Corpus Linguistics, 1, 7-18.

Carroll, B. J. (1961). Fundamental considerations in Language Testing. En Testing the English Proficiency of Foreign Students (pp. 30-40). Washington, DC: Center for Applied Linguistics

Clapham, C. (1996). The Development of IELTS. A Study of the Effect of Background Knowledge on Reading Comprehension. En M. Milanovic (Ed.), Studies in Language Testing. Cambridge: Cambridge University Press.

Gallardo Pauls, B. (2005). Categorías inferenciales en pragmática clínica. Revista de Neurología, 41(1), 65-71.

ELIA 16, 2016, pp. 79-102ＤOI: http://dx.doi.org/10.12795/elia.2016.i16.04 
James, C. (1998). Errors in language learning and use: Exploring Error Analysis. Londres: Longman.

Johnson, K. (1982). Communicative Syllabus Design and Methodology. Oxford: Pergamon.

Kasper, G. (2000). Data collection in pragmatics research. En H. Spencer-Oatey (Ed.), Culturally speaking (pp. 316-41). New York: Continuum,

Leech, G. (1974). Semantics. The Study of Meaning. Londres: Penguin.

Consejo de Europa (2001). Marco Común Europeo de Referencia para las Lenguas. Bruselas.

Martín Marchante, B. (2015). La evaluación de la competencia pragmática en lengua extranjera a través de una prueba adaptativa. Valencia: Universitat Politècnica de València. Tesis doctoral.

Mestre Mestre, E. M. y Carrió Pastor, M. L. (2012). A pragmatic analysis of errors in University students' writings in English. ESP World, 10, 1- 13.

Mestre Mestre, E. M. y Carrió-Pastor, M. L. (2013a). A proposal for the detection and classification of discourse errors. Procedia Social and Behavioral Sciencies, 95, 528 - 534. https://doi.org/10.1016/j.sbspro.2013.10.678

Mestre-Mestre, E. M. y Carrió-Pastor, M. L. (2013b). A pragmatic perspective to leverage English for specific purposes. Revista Española de Lingüistica Aplicada, 26, $107-120$.

Purpura, J. (2004). Assessing Grammar. Cambridge: Cambridge University Press. https://doi.org/10.1017/CBO9780511733086

Purpura, J. (2009). The Oxford Online Placement Test: What does it measure and how? https://www.oxfordenglishtesting.com/uploadedfiles /6_New_ Look_and_Feel/ Content /oopt_measure .pdf [Fecha de acceso: $26-04-$ 2016]

Roever,C.(2006).Validation ofaweb-basedtestofESL pragmalinguistics. Language Testing, 23 (2), 229-256. https://doi.org/10.1191/02655322061t329oa

Roever, C. (2011). Testing of second language pragmatics: Past and future. Language Testing, 28(4), 463-481. https://doi.org/10.1177/0265532210394633

Rose, K. R. y Kasper, G. (2001). Pragmatics in Language teaching. Cambridge: Cambridge University Press.https://doi.org/10.1017/CBO9781139524797

ELIA 16, 2016, pp. 79-102～DOI: http://dx.doi.org/10.12795/elia.2016.i16.04 
Savignon, S. (1972). Teaching for communicative competence: a research report. Audiovisual Language Journal, 10(3), 153-62.

Searle, J. (1969). Speech acts: An essay in the philosophy of language. Cambridge: Cambridge University Press. https://doi.org/10.1017/ $\underline{\mathrm{CBO} 9781139173438}$

Walters, F. S. (2007). A conversation-analytic hermeneutic rating protocol to assess L2 oral pragmatic competence. Language Testing, 24(2), 155-183. https://doi.org/10.1177/0265532207076362

Widdowson, H. G. (1978). Teaching language as communication. Oxford: Oxford University Press.

Yule, G. (1996). Pragmatics. Oxford: Oxford University Press.

Anejo 1. Cuestionario de valoración retrospectivo.

\section{CUESTIONARIO}

Algunos de los motivos por los que has cometido cada error en las preguntas de la prueba de nivel on line pueden ser los siguientes:
A) Lapsus, bloqueo, nervios o prisa
B) No entendí nada
C) Hubo una o dos palabras que no entendí
D) Hubo tres o más palabras que no entendí
E) Me resultó ambiguo; interpreté mal aunque entendí todas las palabras
F) Otros

Por favor en la parrilla que aparece a continuación, señala con una cruz la celda correspondiente al motivo por el que has fallado en algunas de las preguntas de la prueba on line. Solo puedes señalar un motivo. Las preguntas aparecen numeradas de la 11 a la 20 :

ELIA 16, 2016, pp. 79-102～DOI: http://dx.doi.org/10.12795/elia.2016.i16.04 


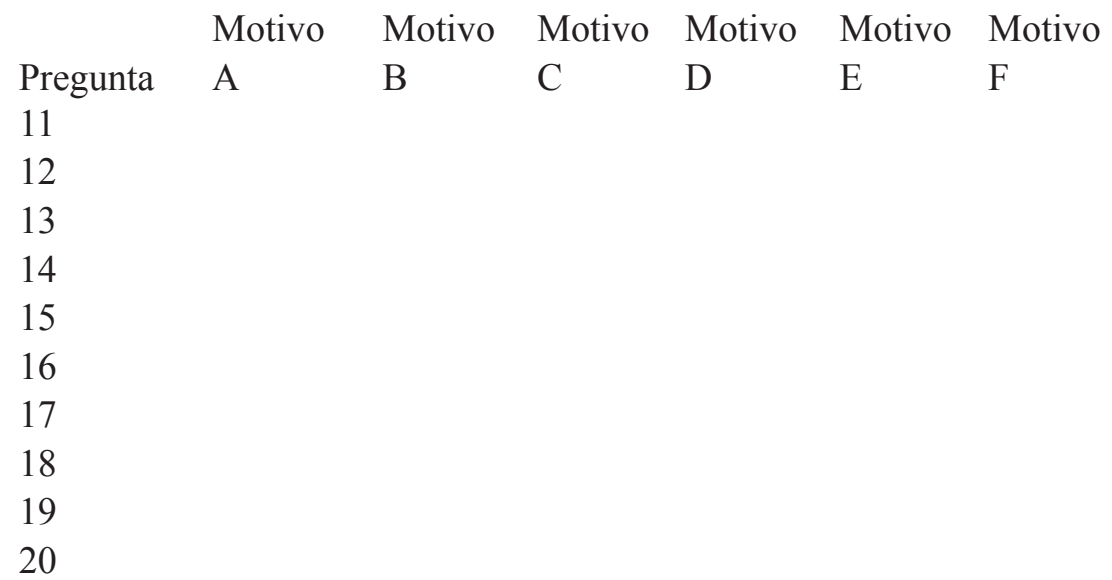

Comentarios sobre el test y las posibles causas de errores:

Anejo 2. Ejemplos de la pregunta 15, realizada incorrectamente por distintos participantes:

Ejemplo 15.1

Mother: Have you learnt all your lines for the play tonight?

Son: $\quad$ Yes, but I know I'll forget them as soon as I get on the stage.

Mother: $\quad$ Don't worry, you'll be fine.

What does the son mean?
A I'll be nervous on stage tonight.
B I can't remember my lines now.
C I need to get on the stage as soon as possible.

Ejemplo 15.2

Daughter: I don't understand tonight's history homework.

Father: Why don't you ask John about it?

ELIA 16, 2016, pp. 79-102 DOI: http://dx.doi.org/10.12795/elia.2016.i16.04 
What does the father mean?
A I think John wants to help you with the homework.
B I don't think John understands the homework.
C I think John can help you.

Ejemplo 15.3

Woman: I just don't know what's the matter with me. My boss has invited me to a Paris fashion show and I'm struggling to get excited about it.

Man: You'd normally go without a second thought.

What does the woman mean?
A She is disappointed about the location of the fashion show.
B She is surprised that she was chosen for the business trip.
C She is concerned about her reaction to her boss's invitation.

Ejemplo 15.4

Interviewer: You've had so many of your plays staged in London that I'm afraid I've lost count.

Playwright: True, but initially, my first play didn't go down very well with the producers who read it.

Interviewer: And then, against all the odds, came the big chance!

What does the interviewer mean by his SECOND comment?

A You had a chance to succeed that you had not expected.

B You took your chance when the opportunity came.

C You got the chance you deserved.

First version received: June 2016

Final version accepted: September 2016

ELIA 16, 2016, pp. 79-102～DOI: http://dx.doi.org/10.12795/elia.2016.i16.04 